\title{
Reduction of hyperthermia in pediatric patients with severe traumatic brain injury: a quality improvement initiative
}

\author{
Marlina E. Lovett, MD, ${ }^{1,2}$ Melissa Moore-Clingenpeel, MA, MAS, ${ }^{3}$ Onsy Ayad, MD, ${ }^{1,2}$ and \\ Nicole O'Brien, MD'1,2 \\ 1Division of Critical Care and 'Biostatistics Core, The Research Institute, Nationwide Children's Hospital; and 2Department of \\ Pediatrics, The Ohio State University College of Medicine, Columbus, Ohio
}

\begin{abstract}
OBJECTIVE Severe traumatic brain injury remains a leading cause of morbidity and mortality in the pediatric population. Providers focus on reducing secondary brain injury by avoiding hypoxemia, avoiding hypotension, providing normoventilation, treating intracranial hypertension, and reducing cerebral metabolic demand. Hyperthermia is frequently present in patients with severe traumatic brain injury, contributes to cerebral metabolic demand, and is associated with prolonged hospital admission as well as impaired neurological outcome. The objective of this quality improvement initiative was to reduce the duration of hyperthermia for pediatric patients with severe traumatic brain injury during the initial 72 hours of admission to the pediatric intensive care unit.
\end{abstract}

METHODS A retrospective chart review was performed to evaluate the incidence and duration of hyperthermia within a preintervention cohort. The retrospective phase was followed by three 6-month intervention periods (intervention Phase 1, the maintenance phase, and intervention Phase 2). Intervention Phase 1 entailed placement of a cooling blanket on the bed prior to patient arrival and turning it on once the patient's temperature rose above normothermia. The maintenance phase focused on sustaining the results of Phase 1. Intervention Phase 2 focused on total prevention of hyperthermia by initiating cooling blanket use immediately upon patient arrival to the intensive care unit.

RESULTS The median hyperthermia duration in the preintervention cohort $(n=47)$ was 135 minutes. This was reduced in the Phase 1 cohort $(n=9)$ to 45 minutes, increased in the maintenance phase cohort $(n=6)$ to 88.5 minutes, and decreased again in the Phase 2 cohort $(n=9)$ to a median value of 0 minutes. Eight percent of patients in the intervention cohorts required additional sedation to tolerate the cooling blanket. Eight percent of patients in the intervention cohorts became briefly hypothermic while on the cooling blanket. No patient required neuromuscular blockade to tolerate the cooling blanket, experienced an arrhythmia, had new coagulopathy, or developed a pressure ulcer.

CONCLUSIONS The placement of a cooling blanket on the bed prior to patient arrival and actively targeting normothermia successfully reduced the incidence and duration of hyperthermia with minimal adverse events.

https://thejns.org/doi/abs/10.3171/2017.8.PEDS17104

KEY WORDS quality improvement; brain injury; pediatric intensive care; hyperthermia reduction; trauma

$\mathrm{S}$ EVERE traumatic brain injury (TBI) remains a leading cause of mortality in the pediatric population, ${ }^{4}$ and survivors of severe TBI can be left with substantial morbidity. Unfortunately, there is no way to reverse damage from the primary insult, and providers can only focus on reducing secondary brain injury. Secondary brain injury is the result of molecular mechanisms that begin at the time of the initial injury but persist and can progress over the following days. Contributors to secondary brain injury include factors such as ischemia, edema, the inflam- matory process, neuronal apoptosis, metabolic dysfunction, and neuronal excitotoxicity. ${ }^{17}$ Therefore, in an attempt to minimize these insults, postinjury management focuses on avoiding hypoxemia and hypotension, providing normoventilation, decreasing cerebral metabolic demand, and treating intracranial hypertension. ${ }^{10}$

Hyperthermia after severe TBI is common, with both a varying definition $\left(\geq 38^{\circ} \mathrm{C}\right.$ or $\left.38.5^{\circ} \mathrm{C}\right)$ and incidence rate $(30 \%-80 \%)$ reported in the literature..$^{1,2,9}$ The etiology of hyperthermia is multifactorial and has been attributed to 


\section{Specific Aim}

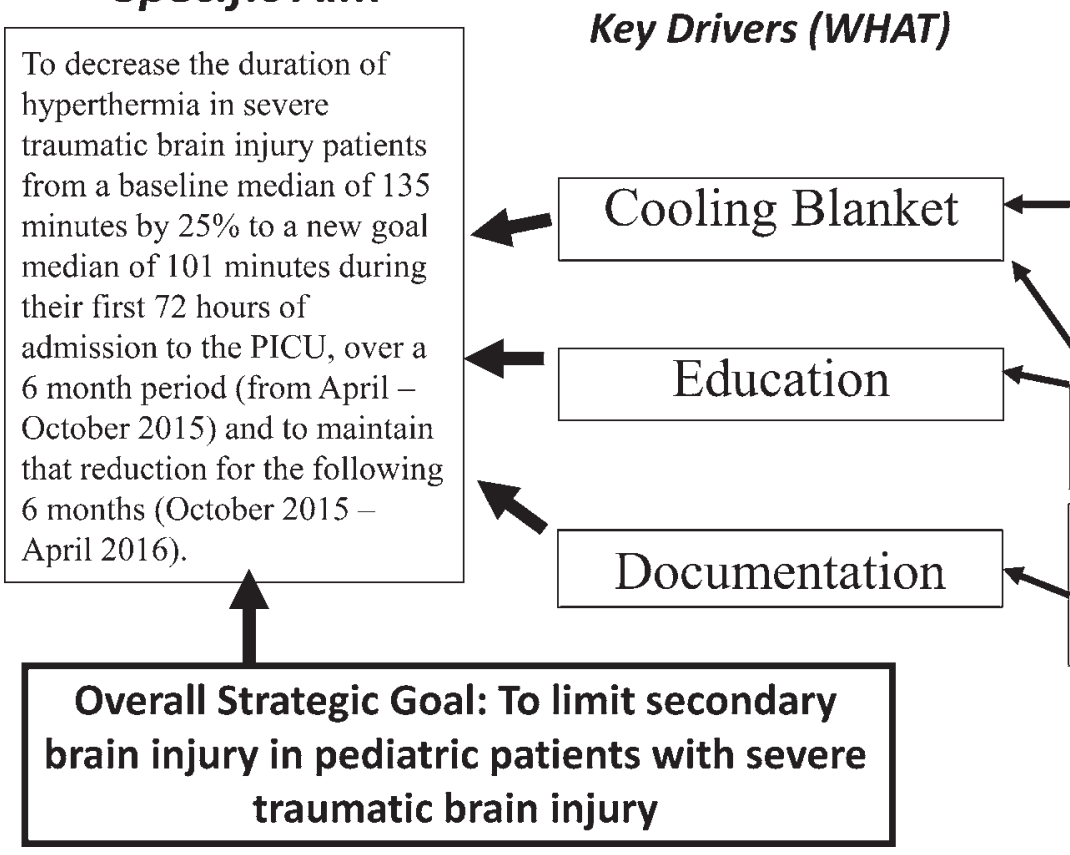

Design Changes or Interventions (HOW)

Ensure timely use of the cooling blanket

$>$ Add to the trauma sheet and specify to be used for all Level 1 Neuro patients

$\checkmark$ Reminder on in-room computers to fill out the trauma sheet

$>$ Try to add cooling blanket to the trauma order set

Educate bedside staff and MDs regarding the impact of hyperthermia on secondary brain injury

Ensure $\mathrm{q} 1 \mathrm{~h}$ vitals are ordered on all severe TBI patients.

Ensure a rectal probe is used while the cooling blanket is being used.

FIG. 1. Key driver diagram to reduce hyperthermia in pediatric patients with severe TBI. $\mathrm{q}^{1 \mathrm{~h}}=$ once every hour.

neuronal excitotoxicity, catecholamine release, the inflammatory response, disruption of the blood-brain barrier, intraparenchymal blood, and alteration of the hypothalamic set point. ${ }^{2}$ Hyperthermia increases cerebral metabolic demand, contributes to intracranial hypertension, and leads to secondary brain injury. ${ }^{2,6,7}$ In animal models with controlled brain injury (a model of complete cerebral ischemia), increases in temperature of $1{ }^{\circ} \mathrm{C}$ were associated with greater histopathological evidence of neuronal injury as well as impaired neurological function. ${ }^{16}$ Clinically, hyperthermia has been associated with a prolonged intensive care unit (ICU) length of stay, poor neurological outcome, and an increased risk of TBI-related mortality. ${ }^{3,5,8,11,13} \mathrm{In}$ addition, studies have shown that a reduction of hyperthermia to normothermia can reduce cerebral oxygen demand in the nonshivering patient?

In an effort to decrease hyperthermia burden in our pediatric ICU (PICU), we implemented a quality improvement initiative targeting the maintenance of normothermia for the first 72 hours of admission following severe TBI. We hypothesized that, if a cooling blanket was placed on the bed prior to patient arrival, then the duration of hyperthermia $\left(\geq 38^{\circ} \mathrm{C}\right)$ would be decreased by $25 \%$. Furthermore, we hypothesized that this reduction would occur over a 6-month implementation period and that the effect would be sustained for an additional 6 months. We also hypothesized that in Phase 2 of our intervention, we would be able to reduce both hyperthermia incidence and duration by an additional $25 \%$ from the prior phase.

\section{Methods}

This project was conducted within the PICU of a pediatric quaternary referral hospital within a Level 1 trauma center. This protocol was evaluated by our institutional re- view board and determined to be a quality improvement project and, as such, did not require institutional review board review or patient informed consent.

A retrospective chart review was performed to evaluate the incidence and duration of hyperthermia in our pediatric TBI population from February 2011 to March 2015 (the period preceding initiation of our project). Inclusion criteria were as follows: 1 ) age $0-18$ years; 2 ) severe TBI as defined by postresuscitation Glasgow Coma Scale (GCS) score $\leq 8 ; 3$ ) abnormal findings on head imaging (presence of intraparenchymal and/or intracranial but extraaxial hemorrhage or signs of diffuse axonal injury); and 4) intubation prior to PICU arrival. There were no exclusion criteria. Variables extracted from the medical record for all patients included patient age, mechanism of injury, postresuscitation GCS score (initial GCS score upon PICU arrival), initial temperature, peak temperature, hyperthermia duration for all temperatures $\geq 38^{\circ} \mathrm{C}$, documentation of cooling blanket use, neurosurgical interventions, acetaminophen/ibuprofen use in the first 72 hours of admission, and ICU length of stay. This initial data extraction is referred to as the "preintervention period" for the remainder of the article. In addition, because this was a quality improvement project focused on the duration of hyperthermia over a set period, all patients needed to remain in the PICU for a minimum of 72 hours. Therefore, if patients died or were discharged from the PICU within 72 hours, they were excluded from our data analysis. The initial 72hour period was chosen because cerebral edema is known to progress during this time, and reduction of secondary brain injury is paramount.

The primary intervention in Phase 1 focused on placement of an external cooling blanket on the bed prior to patient arrival to the ICU (key driver diagram; Fig. 1). The 
TABLE 1. Characteristics of the pre- and postintervention cohorts during the first 72 hours of admission

\begin{tabular}{|c|c|c|c|c|}
\hline Variable & Preintervention Phase & Intervention Phase 1 & Maintenance Phase & Intervention Phase 2 \\
\hline No. of patients & 47 & 9 & 6 & 9 \\
\hline Median age, yrs & $4.6(1.4-10.8)$ & $9(3-13)$ & $4.5(2.5-11)$ & $8(1.75-9)$ \\
\hline Median GCS score & $6 \mathrm{~T}(4-7)$ & $3 \mathrm{~T}(3-6)$ & $3 \mathrm{~T}(3-3)$ & $3 \mathrm{~T}(3-6)$ \\
\hline Median Injury Severity Score & $26(17-29)$ & $26(17-29)$ & $26(19-29)$ & $26(19-34)$ \\
\hline Median Rotterdam score & $1(0-2)$ & $1(1-1)$ & $2(1.25-2.75)$ & $1(0-1)$ \\
\hline Neurosurgical intervention, no. of patients $(\%)^{*}$ & $32(68)$ & $8(89)$ & $5(83)$ & $4(44)$ \\
\hline Median initial temp, ${ }^{\circ} \mathrm{C}$ & $36.5(35.2-37)$ & $35.9(34.6-36.6)$ & $35.5(35.2-37)$ & $36.3(35.8-36.5)$ \\
\hline Patients febrile, $\geq 38^{\circ} \mathrm{C}$, no. (\%) & $35(74)$ & $7(78)$ & $5(83)$ & $3(33)$ \\
\hline Median fever length, mins & $135(8-462)$ & $45(30-90) ; p=0.29$ & $88.5(49-119) ; p=0.62$ & $0(0-120) ; p=0.04$ \\
\hline Median peak temp, ${ }^{\circ} \mathrm{C}$ & $38.3(38-38.9)$ & $38.1(38-38.2) ; p=0.27$ & $38.3(38-38.5) ; p=0.75$ & $37.9(37.8-38.5) ; p=0.21$ \\
\hline Cooling blankets used, no. of patients (\%) & $31(66)$ & $8(89)$ & $6(100)$ & $8(89)$ \\
\hline Acetaminophen received, no. of patients (\%) & $32(68)$ & $4(44)$ & $2(33)$ & $4(44)$ \\
\hline Ibuprofen received, no. of patients (\%) & 0 & 0 & $1(16)$ & 0 \\
\hline Mortality rate & $15 \%$ & $11 \%$ & $33 \%$ & $33 \%$ \\
\hline Median PICU LOS, hrs & $211(139-355)$ & $229(189-427)$ & $218(115-328)$ & $236(95-313)$ \\
\hline Median hospital LOS, hrs & $907(533-1938)$ & $1046(497-1852)$ & $994(260-1409)$ & $632(95-901)$ \\
\hline
\end{tabular}

LOS = length of stay; temp = temperature.

All median values are shown with their respective interquartile range. All displayed $p$ values are for the group displayed compared with the preintervention cohort.

* Neurosurgical interventions included intracranial pressure monitoring, external ventricular drain placement, and craniectomy.

servo-controlled, temperature-regulated cooling blanket (Blanketrol II or Blanketrol III, Cincinnati Sub-Zero) was not turned on at the time of arrival but when the patient's temperature increased above normothermia $\left(36^{\circ} \mathrm{C}-\right.$ $\left.37.5^{\circ} \mathrm{C}\right)$. The cooling blanket was placed in auto or gradient mode. Both modes adjust the water temperature based on the patient's core temperature (which, in this project, was monitored via a bladder or rectal temperature probe).

Complications from the use of the external cooling blanket (hypothermia defined as temperature $<35^{\circ} \mathrm{C}$, shivering, arrhythmias, coagulopathy, and pressure ulcers), PICU length of stay, and hospital length of stay were noted. After multidisciplinary education was complete, we prospectively observed all pediatric patients with severe TBI admitted to the PICU between April 1, 2015, and October 1, 2015 (intervention Phase 1 cohort), and gave feedback to providers in real time.

A maintenance phase included patients who met inclusion criteria in the 6 months after intervention Phase 1 (from October 2, 2015, to April 2, 2016). Prospective data collection included the same variables recorded during the retrospective period as well as complications associated with use of the cooling blanket. During intervention Phase 2 , the temperature-regulated cooling blanket was turned on at the time of patient arrival to prevent any period of hyperthermia rather than turning it on only when the patient's temperature rose above the normothermia range. In addition, a unit-based normothermia guideline was implemented as a nursing resource, which recommended using 2 cooling blankets (1 anterior and 1 posterior) if the patient was persistently hyperthermic following initiation of a single cooling blanket. This recommendation was present in case the patient had a body surface area exceeding that covered by a single cooling blanket (e.g., an adolescent). This 6-month phase was from April 3, 2016, to October 31, 2016.
Within our institution, patients with severe TBI are comanaged by the critical care team and the neurosurgery team. There is an institutional protocol for the treatment of intracranial hypertension, which includes avoidance of hypoxemia; avoidance of aggressive hyperventilation (partial pressure of carbon dioxide goals of 35-40 mm $\mathrm{Hg}$ ); avoidance of hypotension; normoglycemia; normal sodium at a minimum, with frequent use of hyperosmolar therapy; and rapid protocolized treatment of seizures. The ventilator temperature is set to a relative humidity of $37^{\circ} \mathrm{C}$. While the cooling blanket is in use, daily blood cultures are obtained. Measured temperature frequency is every 15 minutes while the cooling blanket is in use, and otherwise every hour. Complete blood counts and electrolyte checks are per the discretion of the treating physician.

Statistical analysis was performed using nonparametric tests, either Mann-Whitney U-test or Fisher's exact test when appropriate. All analysis was performed using Microsoft Excel or GraphPad Prism 7.

\section{Results}

\section{Baseline Data Collection}

During the retrospective data period, 47 patients with severe TBI met inclusion criteria and were in the PICU for a minimum of 72 hours. The median GCS score was 6T ( $\mathrm{T}$ denotes an intubated patient). ${ }^{15}$ The incidence of hyperthermia within the cohort was $74 \%$, and the median duration was 135 minutes. The median peak temperature was $38.3^{\circ} \mathrm{C}$. Cooling blankets were documented as used in $66 \%$ of patients (Table 1 ).

\section{Intervention Phase 1 Data Collection}

During Phase 1, 9 patients with severe TBI met inclu- 
TABLE 2. Complications associated with use of cooling blanket

\begin{tabular}{lccc}
\hline & \multicolumn{3}{c}{ No. of Complications (\%) } \\
\cline { 2 - 4 } \multicolumn{1}{c}{ Complication } & $\begin{array}{c}\text { Intervention } \\
\text { Phase 1, } \\
\mathrm{n}=9\end{array}$ & $\begin{array}{c}\text { Maintenance } \\
\text { Phase, } \\
\mathrm{n}=6\end{array}$ & $\begin{array}{c}\text { Intervention } \\
\text { Phase 2, } \\
\mathrm{n}=9\end{array}$ \\
\hline $\begin{array}{l}\text { Documentation of addi- } \\
\text { tional sedation require- } \\
\text { ments due to shivering }\end{array}$ & $1(11)$ & $1(17)$ & 0 \\
\hline $\begin{array}{l}\text { New neuromuscular block- } \\
\text { ade due to shivering }\end{array}$ & 0 & 0 & 0 \\
\hline New coagulopathy & 0 & 0 & 0 \\
\hline Arrhythmia & 0 & 0 & 0 \\
\hline Pressure ulcers & 0 & 0 & 0 \\
\hline Hypothermia $\left(<35^{\circ} \mathrm{C}\right)$ & 0 & $1(17)$ & $1(11)$ \\
\hline
\end{tabular}

sion criteria and were in the PICU for a minimum of 72 hours. The median GCS score was 3T. Cooling blankets were used for $89 \%$ of patients during this phase. The incidence of hyperthermia among patients was similar to that of the preintervention cohort (78\%). The median peak temperature was $38.1{ }^{\circ} \mathrm{C}$ and was not significantly different from that of the preintervention cohort. However, the median duration of hyperthermia was reduced by $>50 \%$ compared with that of the preintervention cohort, from 135 to 45 minutes (Table 1). One adolescent in Phase 1 had a prolonged period of hyperthermia (915 minutes). Prior to arrival of this patient, the cooling blanket was on the bed, but additional sheets were placed on top of the cooling blanket (between the actual cooling blanket and the patient, which can cause reduced efficacy of the cooling blanket). Due to additional injuries, it was not possible to move or turn this patient to remove excess blankets.

No patient in Phase 1 became hypothermic or developed an arrhythmia, new coagulopathy, or new pressure ulcer (Table 2). One patient in the cohort did require additional sedation for shivering (a single bolus dose of his hourly midazolam [ $4 \mathrm{mg}$, approximately $0.05 \mathrm{mg} / \mathrm{kg}]$ ), although he was not hypothermic at the time. No patients required neuromuscular blockade to tolerate use of the cooling blanket. PICU length of stay was similar between the preintervention and Phase 1 cohorts (Table 1).

\section{Maintenance Phase Data Collection}

The maintenance phase cohort consisted of 6 patients with a median GCS score of 3T. Eighty-three percent of patients experienced hyperthermia within 72 hours, with a median fever duration of 88.5 minutes and a median peak temperature of $38.3^{\circ} \mathrm{C}$. One adolescent in this cohort was hyperthermic for 735 minutes. This patient had a large body surface area and used only 1 cooling blanket, which was inadequate for temperature regulation. One patient required additional sedation for shivering while using the cooling blanket, although he was not hypothermic at the time. The patient shivered when continuous sedative infusions were transitioned from remifentanil alone to fentanyl and midazolam. The patient received an additional 1-time bolus of the hourly rate of both fentanyl $(1 \mu \mathrm{g} / \mathrm{kg})$ and midazolam $(0.1 \mathrm{mg} / \mathrm{kg})$, and his hourly rate of fentanyl was increased from $1 \mu \mathrm{g} / \mathrm{kg} / \mathrm{min}$ to $1.5 \mu \mathrm{g} / \mathrm{kg} / \mathrm{min}$.

In addition, 1 patient became hypothermic while the cooling blanket was in use. The patient had been normothermic until leaving the PICU for brain MRI. Upon return to the PICU, the patient's temperature was $34.5^{\circ} \mathrm{C}$. It was documented that use of the cooling blanket was reinitiated at the time of the patient's return to the PICU after undergoing MRI. The patient was ultimately hypothermic for 75 minutes. It was unclear from the documentation whether the patient was hypothermic prior to reinitiation of use of the cooling blanket. This patient did not require any additional intervention to return to normothermia. There were no other adverse events during this period (Table 2).

\section{Intervention Phase 2}

During intervention Phase 2, the cooling blanket was placed on the patient's bed prior to arrival. Its use was initiated immediately upon the patient's arrival, with the aim to prevent hyperthermia altogether. This is in contrast to the prior phases, where use of the cooling blanket was not initiated until the patient's temperature rose above normothermia. In addition, we recommended the use of cooling blankets both under and over the patient if he/she was persistently hyperthermic (for $>1$ hour with a single cooling blanket). Over this 6-month window, 9 patients with a median GCS score of 3T met inclusion criteria and were in the PICU for a minimum of 72 hours. The incidence of hyperthermia was reduced from $83 \%$ in the maintenance phase to $33 \%$, with a median hyperthermia duration of 0 minutes (interquartile range $0-120$ minutes). During this period, 1 patient was briefly hypothermic while on the cooling blanket for a period of 15 minutes, with a temperature decrease to $34.6^{\circ} \mathrm{C}$. The patient did not require any additional intervention to return to normothermia. There were no other adverse events during this period (Table 2).

\section{Data Analysis}

Compliance with the normothermia protocol was high; the external cooling blanket was on the bed prior to patient arrival in $89 \%$ of Phase 1 patients, $100 \%$ of maintenance phase patients, and $89 \%$ of Phase 2 patients. The median duration of hyperthermia in the preintervention cohort was 135 minutes, which was reduced to 45 minutes during intervention Phase 1 , and even further reduced to 0 minutes in the Phase 2 cohort, which was statistically significant $(p=0.04)$. There was significantly less acetaminophen administered in the intervention and maintenance phases than in the preintervention group $(p=0.04)$. There were no statistically significant differences between the groups in terms of neurosurgical intervention, mortality, or lengths of stay (Table 1). However, the sample size was small, and the study probably lacked power to determine such a difference. A run chart is shown in Fig. 2, depicting the impact of the normothermia interventions.

To better understand the relationship between hyperthermia incidence/duration and factors such as age and head imaging, additional data analysis was performed. Rotterdam scores were compared between the children who had hyperthermia and those who did not. There were 


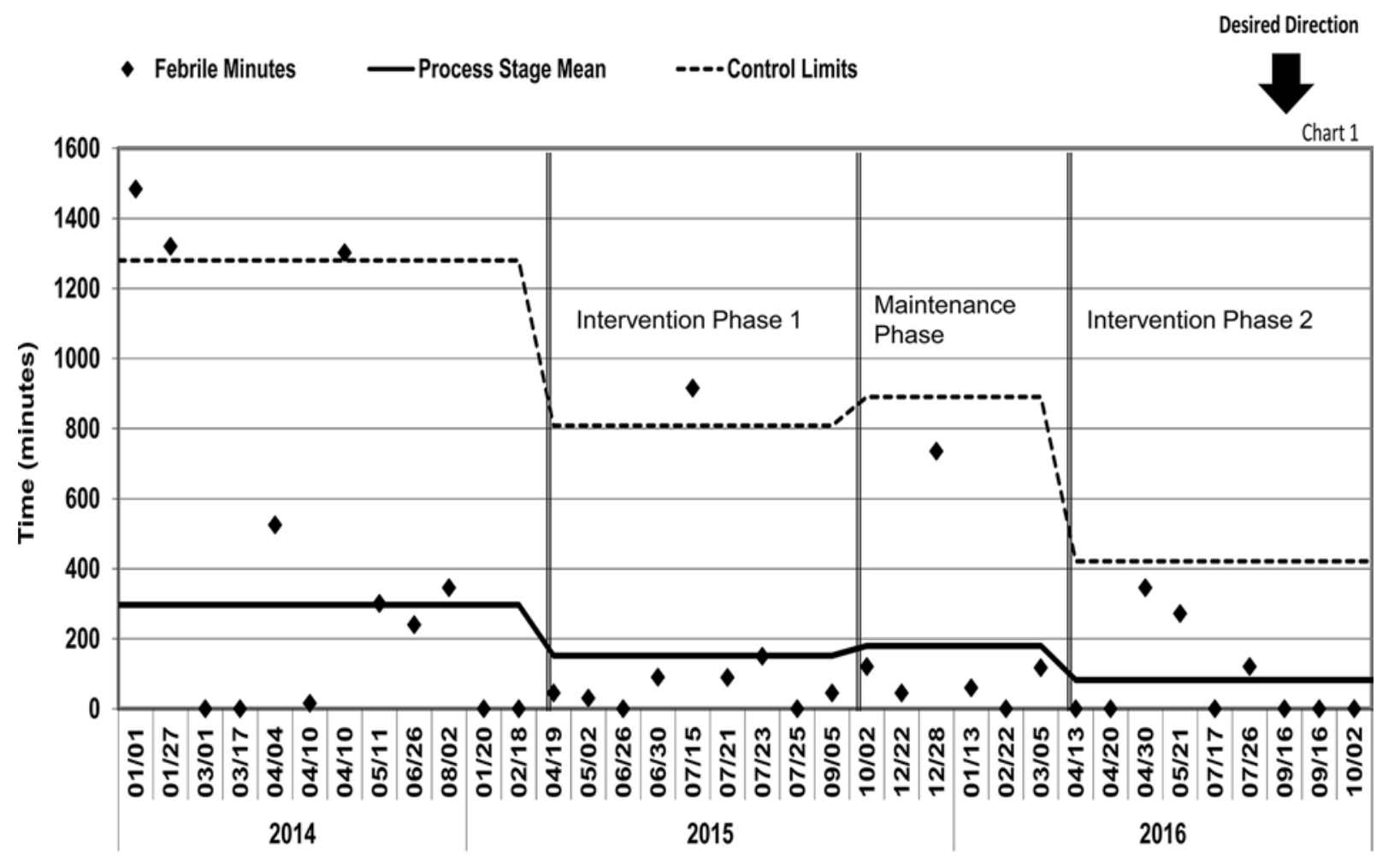

FIG. 2. Run chart showing initiation of the normothermia protocol. The diamonds represent the amount of time that each patient spent febrile. The solid black line denotes the mean time of fever for each cohort.

no statistically significant differences between the groups. To evaluate the impact of age, the median age of those who experienced hyperthermia was compared with that of those who did not. The median age of those with hyperthermia (preintervention, maintenance phase, and intervention Phase 2 cohorts) was 6.75 years $(n=50)$ versus 4 years in the group without hyperthermia $(n=21)$; this difference was not statistically significant. However, when evaluating solely the intervention cohorts (intervention Phase 1, maintenance phase, and intervention Phase 2), the median age of those with hyperthermia was 9 years $(\mathrm{n}=$ $15)$ versus 2 years for those without hyperthermia $(n=9)$; this was statistically significant $(\mathrm{p}=0.02)$.

\section{Discussion}

The incidence of hyperthermia $\geq 38^{\circ} \mathrm{C}$ was $74 \%$ in the retrospective portion of this project. This is similar to the incidence reported in the literature. ${ }^{1,2,9}$ Because hyperthermia can contribute to secondary brain injury, there is a strong impetus to reduce its occurrence in an effort to improve patient outcomes. Given that cerebral edema progresses during the first 72 hours of admission, we believed that it was appropriate to focus on primary reduction of hyperthermia during this initial period.

This quality improvement project was broken down into three 6-month phases. Phase 1 focused on placement of the cooling blanket on the bed prior to patient arrival, use of the cooling blanket once the patient's temperature was higher than the normothermia target, and staff education. The aim of the maintenance phase was solely to ensure that the results of Phase 1 were continued. However, during this phase, the duration of hyperthermia was more prolonged than in patients in Phase 1. Upon review of patients with prolonged hyperthermia, it was thought that using only 1 cooling blanket was insufficient to reduce hyperthermia in certain patients, such as an adultsized adolescent.

Therefore, in Phase 2, a unit-based normothermia guideline was implemented, which included a recommendation to consider an additional cooling blanket if the patient was considered to be too large for a single blanket (suggested for patients $\geq 8$ years of age, based on our institutional experience) or was persistently febrile (for $>1$ hour). For these children, we recommend placement of 1 cooling blanket underneath the patient and 1 on top of the patient. During Phase 2, we transitioned to using the cooling blanket in auto or gradient mode at the time of patient arrival as opposed to when the patient was no longer normothermic, in an attempt to prevent hyperthermia altogether.

There are multiple methods to reduce fever, and when developing a protocol to address hyperthermia, the adverse effects of each method must be considered. Intravascular cooling devices have been successfully used in other institutions. ${ }^{12}$ However, such devices require the placement of a specific central line that is invasive and not routinely used in our institution. Pharmacological methods (e.g., acetaminophen) for fever reduction are routinely used in the reduction of hyperthermia in the ICU. However, the drug's onset of action may be delayed and thus expose the patient to longer durations of hyperthermia than does use of a cooling blanket. In addition, our intervention cohorts 
experienced a shorter duration of hyperthermia despite receiving pharmacological interventions less frequently.

The prospective, intervention portion of this project revealed few adverse effects of the cooling blanket; there were no instances of coagulopathy, arrhythmia, or pressure ulcers. Because the presence of shivering negates the beneficial effect of normothermia on cerebral metabolic demand, it is imperative to closely monitor all patients for shivering.? Two patients (8\%) required increased sedation for shivering, and 2 patients (8\%) experienced a brief period of hypothermia while on the cooling blanket. Given the limited adverse effects of the external cooling blanket, we believe that it can be safely used as first-line management for hyperthermia.

The intervention cohort data suggest that patients with hyperthermia were older (median age 9 vs 2 years). Given their larger body surface area, when these children develop hyperthermia, they may require more intervention and a longer period of time to return to normothermia. Therefore, there may be an additional impetus on the treating clinician to aggressively prevent hyperthermia in the older/larger child. A single cooling blanket may not be adequate. Additional research is needed to provide clinical guidance on how best to prevent and treat hyperthermia in this cohort of patients.

During this project, compliance with our normothermia protocol was evaluated. Every bedside nurse was educated in the protocol. Nursing documentation qualitatively improved, yet it was not possible to effectively measure it. However, our PICU was compliant with both placing the cooling blanket on the bed prior to patient arrival and actively using the cooling blanket. Although these interventions do require additional planning prior to patient arrival, our high compliance rate $(89 \%-100 \%)$ suggests that it is feasible.

This quality improvement project suggests that it is possible to reduce the duration of hyperthermia in the severe TBI population simply by having a cooling blanket on the patient's bed prior to arrival. Although the duration of hyperthermia was successfully reduced, the incidence of hyperthermia remained high. To reduce the incidence of hyperthermia, we began Phase 2, which showed a successful reduction of hyperthermia incidence to $33 \%$. Our unit's focus has changed from solely reducing the duration of fever to eliminating it altogether. Additional future interventions will include using pharmacological methods of fever reduction in a prophylactic and protocolized manner.

Literature suggests that pediatric patients with hyperthermia are more likely to have a lower GCS score at the time of discharge. ${ }^{11,14}$ In adult patients, those with a good recovery 6 months after the initial insult were less likely to have early hyperthermia, whereas those with intermediate outcomes were more likely to have early hyperthermia. ${ }^{6}$ Jiang et al. also found an association between hyperthermia and impaired neurological outcomes via the Glasgow Outcome Scale in populations of both pediatric and predominantly adult patients. ${ }^{8}$

Although these studies have noted an association between hyperthermia and impaired neurological outcomes, correction of hyperthermia has not been proven to improve outcomes. Within our intervention cohort, the hy- perthermia burden was reduced, yet neither PICU nor hospital lengths of stay were shorter. It is possible that as patients are added to the intervention cohort, differences in outcomes will emerge. Therefore, additional research on the long-term neurological implications of hyperthermia reduction within the pediatric population is needed.

This quality improvement project has a number of limitations. Our intervention cohorts were small, and it remains to be seen if the significant differences in fever duration will persist as the cohort size becomes larger. Given the small sample size, the study probably lacked power to detect small differences. Moreover, data available for use were limited to those documented in charts. Therefore, it is possible that some patients may have used a cooling blanket but it was not documented. Similarly, fever length was dependent on nursing documentation. Within our institution, documentation of temperature while the cooling blanket is in use is recommended every 15 minutes. However, if documentation was poor, a patient may have seemed to have had a prolonged period of hyperthermia when actually normothermic. In addition, although we have shown a reduction in the duration and incidence of hyperthermia, we lack neurodevelopmental follow-up data to determine if our intervention had an impact on longterm outcomes.

\section{Conclusions}

Hyperthermia is common within the pediatric population with severe TBI. It is reasonable to target reduction of hyperthermia in an attempt to reduce secondary brain injury. We have shown that it is possible to reduce the incidence and duration of hyperthermia by placing a cooling blanket on the bed prior to patient arrival and by actively targeting normothermia from the time of admission, without causing harm. Additional research is needed to fully investigate the associations among hyperthermia, hyperthermia reduction, and long-term neurological outcome within the pediatric severe TBI population.

\section{Acknowledgments}

We acknowledge the Nationwide Children's Trauma Nurse Resource Group for its assistance with this quality improvement project as well as its continued dedication to providing the best care possible to pediatric trauma patients. We also acknowledge Jessica Dopkiss, RN, for her continued leadership with implementation of this project.

\section{References}

1. Alharfi IM, Charyk Stewart T, Al Helali I, Daoud H, Fraser DD: Infection rates, fevers, and associated factors in pediatric severe traumatic brain injury. J Neurotrauma 31:452-458, 2014

2. Badjatia N: Hyperthermia and fever control in brain injury. Crit Care Med 37 (7 Suppl):S250-S257, 2009

3. Bao L, Chen D, Ding L, Ling W, Xu F: Fever burden is an independent predictor for prognosis of traumatic brain injury. PLoS One 9:e90956, 2014

4. Coronado VG, Xu L, Basavaraju SV, McGuire LC, Wald MM, Faul MD, et al: Surveillance for traumatic brain injuryrelated deaths-United States, 1997-2007. MMWR Surveill Summ 60:1-32, 2011 
5. Diringer MN, Reaven NL, Funk SE, Uman GC: Elevated body temperature independently contributes to increased length of stay in neurologic intensive care unit patients. Crit Care Med 32:1489-1495, 2004

6. Geffroy A, Bronchard R, Merckx P, Seince PF, Faillot T, Albaladejo P, et al: Severe traumatic head injury in adults: which patients are at risk of early hyperthermia? Intensive Care Med 30:785-790, 2004

7. Hata JS, Shelsky CR, Hindman BJ, Smith TC, Simmons JS, Todd MM: A prospective, observational clinical trial of fever reduction to reduce systemic oxygen consumption in the setting of acute brain injury. Neurocrit Care 9:37-44, 2008

8. Jiang JY, Gao GY, Li WP, Yu MK, Zhu C: Early indicators of prognosis in 846 cases of severe traumatic brain injury. $\mathbf{J}$ Neurotrauma 19:869-874, 2002

9. Johnston NJ, King AT, Protheroe R, Childs C: Body temperature management after severe traumatic brain injury: methods and protocols used in the United Kingdom and Ireland. Resuscitation 70:254-262, 2006

10. Kochanek PM, Carney N, Adelson PD, Ashwal S, Bell MJ, Bratton $S$, et al: Guidelines for the acute medical management of severe traumatic brain injury in infants, children, and adolescents - second edition. Pediatr Crit Care Med 13 (Suppl 1):S1-S82, 2012

11. Natale JE, Joseph JG, Helfaer MA, Shaffner DH: Early hyperthermia after traumatic brain injury in children: risk factors, influence on length of stay, and effect on short-term neurologic status. Crit Care Med 28:2608-2615, 2000

12. Puccio AM, Fischer MR, Jankowitz BT, Yonas H, Darby JM, Okonkwo DO: Induced normothermia attenuates intracranial hypertension and reduces fever burden after severe traumatic brain injury. Neurocrit Care 11:82-87, 2009

13. Saxena M, Young P, Pilcher D, Bailey M, Harrison D, Bellomo R, et al: Early temperature and mortality in critically ill patients with acute neurological diseases: trauma and stroke differ from infection. Intensive Care Med 41:823-832, 2015

14. Suz P, Vavilala MS, Souter M, Muangman S, Lam AM: Clinical features of fever associated with poor outcome in severe pediatric traumatic brain injury. J Neurosurg Anesthesiol 18:5-10, 2006
15. Teasdale G, Jennett B: Assessment of coma and impaired consciousness. A practical scale. Lancet 2:81-84, 1974

16. Wass CT, Lanier WL, Hofer RE, Scheithauer BW, Andrews AG: Temperature changes of $>$ or $=1$ degree $C$ alter functional neurologic outcome and histopathology in a canine model of complete cerebral ischemia. Anesthesiology 83:325-335, 1995

17. Werner C, Engelhard K: Pathophysiology of traumatic brain injury. Br J Anaesth 99:4-9, 2007

\section{Disclosures}

The authors report no conflict of interest concerning the materials or methods used in this study or the findings specified in this paper.

\section{Author Contributions}

Conception and design: Lovett, Ayad, O'Brien. Acquisition of data: Lovett. Analysis and interpretation of data: Lovett, MooreClingenpeel, O'Brien. Drafting the article: Lovett. Critically revising the article: Lovett, Ayad, O'Brien. Reviewed submitted version of manuscript: all authors. Approved the final version of the manuscript on behalf of all authors: Lovett. Statistical analysis: Lovett, Moore-Clingenpeel.

\section{Supplemental Information \\ Previous Presentations}

Partial data from this study were presented in poster form at the Pediatric Trauma Society's 2nd Annual Meeting, Scottsdale, AZ, November 5, 2015.

\section{Correspondence}

Marlina E. Lovett, Division of Pediatric Critical Care Medicine, Nationwide Children's Hospital, 700 Children's Dr., Columbus, OH 43205. email: marlina.lovett@nationwidechildrens.org. 